\title{
Comment les protéines membranaires sont-elles "ciblées " dans les cellules tubulaires rénales en culture?
}

Dans les cellules épithéliales polarisées, la distribution de la $\mathrm{Na}^{+}, \mathrm{K}^{+}$ATPase est restreinte au domaine membranaire apical, par exemple dans les plexus choroïdes et l'épithélium pigmenté de la rétine, ou à la membrane basolatérale, par exemple dans le rein et l'intestin. Au cours de la différenciation, la $\mathrm{Na}^{+}, \mathrm{K}^{+}$-ATPase est répartic de manière diffuse dans les cellules précurseurs puis cst secondairement localisée au niveau du domaine basolatéral. Le mécanisme qui détermine la localisation de la $\mathrm{Na}^{+}$, $\mathrm{K}^{+}$-ATPase, a été étudié par Hammerton et al. (Stanford et Philadelphie CA et PA, USA) sur des cellules épithéliales de rein de chien Madin-Darby (MDCK) en culture ; à confluence, ces cellules forment des jonctions serrées et acquièrent des fonctions spécifiques des cellules épithéliales polarisées comme cela est observé au cours de la différenciation : dans les 48 premières heures de contact cellulaire, la $\mathrm{Na}^{+}$, $\mathrm{K}^{+}$-ATPase est détectée par immunofluorescence au niveau de la membrane apicale et de la membrane basolatérale. Plus tard, la $\mathrm{Na}^{+}, \mathrm{K}^{+}$-ATPase localisée à la membrane basolatérale prédomine largement. Par marquage court à la méthionine ${ }^{35} \mathrm{~S}$ et chasse isotopique, les auteurs ont déterminé que des quantités égales de la sous-unité $\alpha$ de la $\mathrm{Na}^{+}, \mathrm{K}^{+}$-ATPase nouvellement synthétisée sont apportées aux domaines membranaires, apical et basolatéral, à tous les temps testés. Ces résultats suggèrent qu'il n'y a ni " tri " de la $\mathrm{Na}^{+}, \mathrm{K}^{+}$-ATPase dans l'appareil de Golgi, ni routage préférentiel de l'enzyme vers un domaine membranaire. $\mathrm{La} \mathrm{Na}^{+}, \mathrm{K}^{+}$-ATPase apparaît au niveau de ces deux domaines, normalement constituée de ses chaînes $\alpha$ et $\beta$. Cependant, ce complexe protéique au pôle apical de la cellule ne fixe pas l'ouabaïne, indiquant que la pro- téine n'est pas fonctionnclle. Les auteurs ont montré que le temps de résidence est 40 fois plus long pour l'enzyme baso-latérale que pour l'enzyme apicale. La stabilisation de la $\mathrm{Na}^{+}, \mathrm{K}^{+}$-ATPase dépend de sa liaison à un complexe associant des protéines du cytosquelette, l'ankyrine et la foldrine, avec la membrane plasmique ; la membrane apicale des ccllules MDCK étant dépourvue de ces protéines, il est suggéré que la $\mathrm{Na}^{+}$, $\mathrm{K}^{+}$-ATPase, à son niveau, est inactive et rapidement internaliséc [1].

Le mécanisme réglant la polarité de la $\mathrm{Na}^{+}, \mathrm{K}^{+}$-ATPase n'est pas généralisable à l'ensemble des protéines $\mathrm{mcm}$ branaires. Pour d'autres protéines, il existe un système de transport cellulaire spécifique vers un domaine $\mathrm{mcm}$ branaire. Jalal et al. (Montréal, Canada) ont montré que l'cndopeptidase neutre est directement transportée au domaine apical sans transiter par le domaine basolatéral [3]. De même, la protéine desmogléine 1, qui détermine l'adhérence des cellules, est rapidement restreinte à la membrane basolatérale et distribuée de façon élective à ce site.

Les études faites par Boll et al. (New Haven, Stony Book, NY et Chicago IL, USA) sur les mêmes cellules MDCK précisent que le transport des protéines sécrétées (la laminine et les protéoglycanes héparane sulfate) et des protéines transmembranaires (la $\mathrm{Na}^{+}, \mathrm{K}^{+}$ATPase et la VLA intégrine) diffère et s'effectue par des systèmes de transport distincts [2]. Le transport des premières s'effectue le long des microtubules et est inhibé par leur désorganisation par la colchicine ou le nocodazole. En revanche, la désorganisation des microtubules n'inhibe pas le routage cellulaire de la $\mathrm{Na}^{+}, \mathrm{K}^{+}$-ATPase active (liant l'ouabaïne) et de la VLA intégrine, suggé- rant que le transit cellulaire de ces protéines implique un autre système dc transport cellulaire.

Les mécanismes qui contrôlent la distribution, apicalc ou basolatérale, des protéines membranaires sont essentiels pour établir la polarité et la fonction des cellules épithéliales. Certaines maladies, comme la polykystose rénale $(\mathrm{m} / \mathrm{s}$ $n^{\circ} 9$, vol. 6 , p. 904), pourraient résulter d'une mauvaise localisation de certaines protéines membranaires, ou de la modification de leur stabilité au niveau de domaine membranaire où elles sont normalement faiblement présentes, impliquant des changements de l'interaction de ces protéines avec des protéines du cytosquelettc, ou des variations dans la localisation des protéines du cytosquelette.

A. D. J.-P. G.

1. Hammerton RW, Krzeminski KA, Mays $\mathrm{RW}$, et al. Mechanism for regulating cell surface distribution of $\mathrm{Na}^{+}, \mathrm{K}^{+}$-ATPase in polarized epithelial cells. Science 1991; $254: 847-50$. 2. Boll W, Partin JS, Katz IK, et al. Distinct pathways for basolateral targeting of membrane and secretory proteins in polarized epithelial cells. Proc Nall Acad Sci USA 1991; 88: 8592-6. 3. Jalal F, Lemay G, Zollinger M, et al. Neutral endopeptidase, a major brush border protein of the kidney proximal nephron, is directly targeled to the apical domain when expressed in Madin-Darby canine kidney cells. $J$ Biol Chem $1991 ; 266$ : 19826-32. 